\title{
Répercussions des difficultés dans la compétence scripturale d'enseignants du primaire en contexte d'insertion professionnelle ${ }^{1}$
}

\author{
GENEVIÈVE CARPENTIER \\ Université du Québec en Outaouais
}

\section{Résumé}

L'insertion professionnelle des enseignants est problématique dans plusieurs pays, et ce, pour de multiples raisons, dont la lourdeur des tâches professionnelles. Malgré que plusieurs de ces tâches nécessitent l'écriture, certains étudiants en formation initiale ne semblent pas maitriser suffisamment la compétence scripturale qui est requise pour la réalisation des tâches inhérentes au domaine de l'éducation. En nous référant aux différentes dimensions de la compétence scripturale et à la conceptualisation de l'insertion professionnelle selon Vallerand (2008), nous avons tenté de décrire les difficultés dans la compétence scripturale et les répercussions de celles-ci sur les tâches professionnelles et l'insertion professionnelle d'enseignants débutants.

De nombreux chercheurs estiment qu'une majorité des enseignants éprouvent le sentiment d'être débordés par les tâches professionnelles qui leur incombent lors de leur insertion professionnelle (Beckers, 2007; Mukamurera, Bourque, \& Gingras, 2008). Ce sentiment de ne pas suffire à la tâche peut affecter négativement l'insertion professionnelle. Lorsque nous observons la tâche professionnelle enseignante, nous remarquons que l'écriture est requise dans plusieurs tâches comme planifier les activités d'enseignement, corriger spontanément les écrits d'élèves, écrire au tableau, etc. Pourtant, Lefrançois, Laurier, Lazure, \& Claing (2005) mettent en lumière les difficultés dans la compétence scripturale de certains étudiants au baccalauréat en éducation préscolaire et enseignement primaire (BEPEP). Il est possible de penser que des lacunes dans la compétence scripturale puissent entrainer des difficultés dans la réalisation de certaines tâches professionnelles et, ainsi, avoir certaines répercussions sur l'insertion professionnelle. Aucune étude n'a tenté à ce jour d'établir un lien entre les difficultés vécues lors de l'insertion professionnelle et les difficultés dans la compétence scripturale. Nous présentons, dans cet article, la problématique de notre recherche, les différents concepts sur lesquels elle s'appuie, sa méthodologie ainsi que certains résultats. De plus, nous exposons une brève discussion des résultats présentés.

\section{Problématique}

Le passage de la formation initiale vers la pratique, nommé insertion professionnelle, peut s'avérer laborieux, car plusieurs enjeux caractérisent cette période (Martineau \& Vallerand, 2006). Ainsi, les enseignants débutants, qui ont cinq années d'expérience ou moins (Martineau \& Ndoreraho, 2006), peuvent vivre un ou plusieurs problèmes qui rendent leur insertion professionnelle ardue. Ces problèmes ont été

${ }^{1}$ Ce texte adopte l'orthographe rectifiée.

Language and Literacy $\quad$ Volume 16, Issue 2, Special Issue $2014 \quad$ Page 38


largement documentés par la communauté scientifique et les principales difficultés recensées sont notamment des difficultés d'ordre personnel, socioprofessionnel, pédagogique et didactique; des difficultés liées à l'isolement professionnel et à l'absence de collaboration du milieu professionnel enseignant; et des difficultés engendrées par la précarité d'emploi et par le manque d'accompagnement lors de l'insertion professionnelle (Bédard, 2000; Beckers, 2007; Martineau \& Bergevin, 2007; Martineau \& Ndoreraho, 2006; Mukamurera et al., 2008). Ces difficultés peuvent avoir des répercussions négatives pour les enseignants débutants et nous relatons ci-après les conséquences possibles de ces difficultés.

\section{Conséquences de ces difficultés}

La charge de travail élevée peut être une source importante de stress, d'insomnie et de dépression chez les enseignants débutants (Martineau \&_Vallerand, 2005). Dans une étude menée par Mukamurera et al. (2008), $88 \%$ des répondants affirment que la charge de travail élevée est l'obstacle le plus difficile à surmonter lors de l'insertion. En outre, cela peut entrainer un manque de confiance et peut nuire au sentiment d'efficacité professionnelle. De plus, le stress dû à une surcharge de travail nuit à l'enseignement, amplifie l'impatience et diminue le renforcement positif fait aux élèves (Martineau \& Presseau, 2003). Martineau et Mukamurera (2012) affirment que lorsque les problèmes rencontrés par les débutants ne sont pas reconnus et qu'il n'y a pas de moyens concrets mis en place pour les soutenir, il est possible que ces nouveaux enseignants souffrent de problèmes physiques ou psychologiques. L'attrition professionnelle est aussi une conséquence de ces difficultés, et un enjeu de taille, puisque $15 \%$ des enseignants débutants quitteraient la profession au cours de leurs cinq premières années dans la profession. Par ailleurs, plusieurs études mentionnent que les enseignants débutants qui quittent la profession sont souvent les enseignants les plus prometteurs qui vivent des difficultés liées à leur insertion professionnelle (Curtis, 2005). Considérant tous ces impacts potentiels en lien avec une surcharge de travail, il importe de nous pencher sur les tâches professionnelles des enseignants débutants.

\section{Tâches professionnelles}

Selon la Centrale des syndicats du Québec (CSQ, 2005) et la Fédération des syndicats de l'enseignement (FSE, 2011), la tâche professionnelle enseignante est divisée en trois grandes catégories :

1. La tâche éducative (période d'enseignement, récupération, activités parascolaires)

2. La tâche complémentaire (surveillance lors des déplacements, suivis pédagogiques)

3. La tâche personnelle (rencontres de parents, planification des activités d'enseignement, correction et consignation des résultats)

L'usage de l'écriture est requis dans tous les types de tâches: 1) la tâche éducative: écrire au tableau (notes de cours, explications, remue-méninges, etc.), communiquer par écrit avec les parents: lettres, agendas, etc. ; 2) la tâche complémentaire : consignation du suivi pédagogique, écriture des plans d'intervention, 
prises de notes lors des rencontres; 3) la tâche personnelle : rédiger des dictées, corriger spontanément des écrits d'élèves, corriger des situations d'écriture, composer des situations d'apprentissage, etc. (Ministère de l'Éducation du Québec, 2001). Finalement, les tâches reliées à l'écriture peuvent être laborieuses sur le plan grammatical pour les enseignants débutants ayant des difficultés dans la maitrise du français écrit et pourraient peut-être contribuer au sentiment d'être dépassé par la charge de travail.

\section{Difficultés des étudiants dans la compétence scripturale}

La compétence scripturale se définit comme étant « la capacité de produire du sens par l'écrit sans qu'il y ait transit par l'oral, par l'appropriation des outils sémiotiques et matériels de l'écrit; c'est développer le contrôle de son activité langagière en prenant son texte comme un objet qu'on crée, commente, structure, manipule et transforme » (Chartrand, 2000, p. 26). Les résultats et les recommandations de plusieurs recherches et rapports convergent vers la piètre qualité de la compétence scripturale de plusieurs étudiants québécois (Larose, 2001; Lefrançois et al. 2005). En 2001, la Commission des États généraux sur la situation et l'avenir de la langue français au Québec rapporte le niveau de compétence à écrire de 1156 étudiants universitaires des domaines de l'éducation et des sciences qui ont été évalués au moyen de tests diagnostiques. Le rapport de la Commission révèle que ces étudiants manifestaient les difficultés langagières suivantes: $75 \%$ d'entre eux avaient des lacunes en ce qui a trait au vocabulaire, $70 \%$ en conjugaison, $61 \%$ avec les accords des participes passés et $50 \%$ en syntaxe. Ces étudiants universitaires éprouvaient donc des difficultés marquées sur le plan de la compétence scripturale. Pour leur part, Lefrançois et al. (2005) affirment que les 33 étudiants universitaires en difficulté sélectionnés dans le cadre de leur recherche commettaient 23,68 erreurs pour 250 mots lors de la rédaction d'un texte descriptif. Les erreurs recensées se rapportaient à l'orthographe lexicale et grammaticale, à la conjugaison et à la syntaxe. Pourtant, comme nous l'avons précédemment exposé, la compétence scripturale est constamment sollicitée dans les tâches professionnelles des enseignants, et des difficultés liées à cette compétence pourraient les alourdir davantage. Il est donc légitime de se questionner sur les liens possibles entre les difficultés vécues lors de l'insertion professionnelle et les lacunes dans la compétence scripturale des enseignants débutants.

\section{Question de recherche et objectifs spécifiques}

En tenant compte des difficultés engendrées par l'insertion professionnelle, de l'importance de la compétence scripturale dans les tâches professionnelles et des difficultés éprouvées dans cette compétence par des enseignants débutants, nous avons posé la question suivante : quelles sont les répercussions des difficultés déclarées dans la compétence scripturale d'enseignants débutants du primaire lors de leur insertion professionnelle? De cette question découlaient trois objectifs spécifiques, qui sont : 1) identifier les difficultés déclarées éprouvées par des enseignants débutants du primaire dans la compétence scripturale; 2) décrire les tâches professionnelles de ces enseignants qui sont affectées par leurs difficultés déclarées; 3) décrire les répercussions de ces difficultés sur l'insertion professionnelle de ces enseignants. 


\section{Insertion professionnelle}

\section{Cadre théorique}

Bien qu'il n'y ait pas de consensus à l'égard de la définition du concept d'insertion professionnelle, nous avons retenu, dans le cadre de ce projet de recherche, l'idée d'un processus multidimensionnel d'une durée moyenne de cinq ans (Mukamurera Martineau, Bouthiette, \& Ndoreraho, 2013). Plusieurs façons de conceptualiser l'insertion professionnelle coexistent; celle que nous avons retenue est basée sur l'expérience de vie qui met en relief le vécu subjectif des enseignants débutants et qui se divise en quatre composantes (Vallerand, 2008) présentées ci-après.

\section{La construction et la consolidation des savoirs}

Alors que certains apprentissages sont effectués lors de la formation initiale, d'autres sont consolidés en situation réelle (Norman \& Feiman-Nemser, 2005). Ainsi, «peu importe la qualité de la formation universitaire, il restera toujours des aspects que seule la pratique en milieu réel peut combler» (Bédard, 2000, p.9), comme l'apprentissage de la gestion de classe et certaines habiletés pédagogiques telles que choisir les méthodes d'enseignement ou faire une planification annuelle (Simard, 2005). La maitrise de ces habiletés peut s'avérer complexe pour les débutants et affecter leur sentiment d'efficacité, qui est relié à leurs perceptions concernant leurs compétences professionnelles (Martineau \& Vallerand, 2005).

\section{Développement du rapport aux autres}

Les nouveaux enseignants doivent entrer en relation avec les élèves, leurs parents, les collègues et les directions d'école. Le rapport enseignant-élève est très important et une relation positive peut être une source de valorisation et de motivation. La relation avec les parents des apprenants peut être difficile, car certains parents peuvent se montrer critiques à l'égard des recrues (Boucher, 2001), alors que certains nouveaux enseignants entretiennent des jugements à l'endroit des parents (Vallerand, 2008). La relation avec les collègues, quant à elle, peut être fortement influencée par le type de rapport qu'entretiennent les enseignants entre eux. Ainsi, une école peut être un lieu propice aux échanges ou un milieu froid et solitaire. Finalement, la direction peut influencer positivement l'insertion des enseignants nouvellement qualifiés en les conseillant et en étant à l'écoute.

\section{Développement de l'identité professionnelle}

La troisième composante de l'insertion est liée au développement de l'identité professionnelle (Vallerand, 2008). L'identité professionnelle est un processus personnel et dynamique durant lequel les novices réfléchissent sur leur rôle et sur les valeurs qu'ils désirent véhiculer (Presseau \& Martineau, 2007). «En effet, pour évoluer professionnellement et construire son identité, le novice doit avoir la possibilité de réfléchir sur lui-même et sur ses actes professionnels »(Vallerand, 2008, p. 52). Plusieurs facteurs favorisent une identité professionnelle positive: un accueil chaleureux, de bonnes relations avec les pairs, un sentiment d'efficacité professionnelle, tandis que certains facteurs l'influencent négativement : un manque de reconnaissance, un sentiment d'incompétence pédagogique, une surcharge de travail, etc. Alors que la construction 
d'une identité professionnelle positive peut favoriser l'intégration des débutants à leur milieu et leur estime de soi, des difficultés dans le développement de cette identité peuvent influencer négativement la persévérance professionnelle (Presseau \& Martineau, 2007).

\section{Acquisition de la culture institutionnelle}

Enfin, la dernière composante identifiée par Vallerand (2008) concerne l'appropriation de la culture institutionnelle. Il existe deux types de connaissances liés à la culture institutionnelle d'un établissement scolaire. Le premier type fait référence aux connaissances explicites et englobe les horaires de cours, le projet éducatif de l'école et les règles en vigueur. Ce type de connaissances est souvent accessible aux nouveaux enseignants. Le second type de connaissances est moins explicite et n'est habituellement pas présenté aux débutants (Vallerand, 2008). Ces connaissances incluent la philosophie de l'école, les habitudes des collègues, les méthodes pédagogiques préconisées par les pairs, etc. Il peut être difficile pour les débutants de saisir la culture institutionnelle, surtout dans les situations où ils sont appelés à changer d'école fréquemment.

Les composantes de Vallerand (2008), décrites ici, ont servi de cadre d'analyse tout comme les différentes dimensions de la compétence scripturale présentées brièvement dans la section suivante.

Compétence scripturale. Selon Schneuwly (1995), écrire c'est agir, c'est une pratique en mouvement, changeante, préparée et réfléchie. " Agir langagièrement », c'est comprendre que l'acte d'écriture nécessite l'appropriation de l'ordre scriptural, idée reprise par Chartrand (2000) qui mentionne également que l'écriture :

« c'est entrer dans l'ordre scriptural, concept développé par Peytard (1970) et repris et élaboré par Dabène (1987). Ce concept fait référence à la capacité de produire du sens par l'écrit sans qu'il y ait transit par l'oral, par l'appropriation des outils sémiotiques et matériels de l'écrit; c'est développer le contrôle de son activité langagière en prenant son texte comme un objet qu'on crée, commente, structure, manipule et transforme (p. 26). »

Ainsi, tous les écrits ne s'inscrivent pas dans l'ordre scriptural. Par exemple, la retranscription et le listage sont des écrits qui ne requièrent ni les connaissances ni les appropriations spécifiques à l'ordre scriptural. Toutefois, l'écriture d'une planification ou la rédaction d'un message sont des types d'écrits qui engagent le sujet scripteur, car ils requièrent des connaissances non seulement linguistiques, mais aussi sémantiques, textuelles, syntaxiques, des connaissances du processus d'écriture et des représentations de ses capacités. Plusieurs tâches professionnelles du domaine de l'éducation nécessitent un engagement dans l'acte d'écriture, une maitrise de la compétence scripturale.

Aux fins de cette étude, trois dimensions de la compétence scripturale ont été conceptualisées et ont servi de cadre d'analyse : la dimension épistémique, la dimension affective et la dimension axiologique. Ces dimensions, bien que complémentaires, se présentent aussi en tension, la première entrainant le sujet scripteur à vivre un rapport à l'écriture plus intellectualisé, les deux autres, à vivre un rapport à l'écriture davantage marqué par ses expériences personnelles, sa conception de soi comme scripteur et son 
intérêt pour l'écriture. Cette étude a permis de comprendre comment ces dimensions s'articulent les unes aux autres. Nous exposons succinctement ces trois composantes dans les sections suivantes.

\section{Dimension épistémique}

La dimension épistémique de la compétence scripturale introduit différents savoirs propres à l'écriture et la pensée critique du sujet scripteur sur ces savoirs. Cette dimension englobe les savoirs linguistiques (Dabène, 1987) comme l'orthographe, la grammaire, la syntaxe et le lexique. Il comprend également le processus d'écriture (Hayes \& Flower, 1980) qui nécessite la connaissance des différentes étapes du processus rédactionnel (planification, mise en texte, révision). La dimension épistémique renvoie aussi aux savoirs liés aux genres textuels (Schneuwly, 1995). Ainsi, le sujet scripteur doit reconnaitre et savoir utiliser les normes et les particularités des différents types de texte (texte argumentatif, descriptif, explicatif, etc.). Finalement, la fonction épistémique (Chartrand \& Blaser, 2008) évoque les savoirs liés à l'organisation des idées, au développement et à la progression de l'information, et engendre une pensée critique et une construction de connaissance par et dans l'acte d'écriture.

\section{Dimension affective}

Cette dimension concerne l'affectivité, soit la sphère des sentiments du sujet pour l'écriture. Elle influence en partie la quantité d'énergie (temps, fréquence) déployée par le sujet dans l'acte d'écriture (Chartrand \& Blaser, 2008). Deux aspects distincts composent cette dimension : la force d'investissement et le type d'investissement. « La force d'investissement désigne l'intensité de celui-ci, c'est-à-dire que l'écriture peut être fortement, moyennement ou faiblement investie et valorisée » (Barré-De Miniac, 2000, p. 118). La force d'investissement peut aussi être influencée par le second aspect de cette dimension : le type d'investissement qui représente le genre de texte devant être écrit. Par exemple, l'investissement pour l'enseignant peut être plus grand dans la rédaction d'une note de félicitations à l'agenda que lors de la rédaction d'un examen de lecture.

\section{Dimension axiologique}

Finalement, la dimension axiologique fait référence aux opinions (se rapportant aux dires) et aux attitudes (se rapportant aux comportements) du sujet scripteur par rapport à l'écriture (Chartrand \& Blaser, 2008). Ces opinions et ces attitudes expriment la valeur du sujet envers l'écrit et, lorsqu'elles sont positives, permettent au sujet scripteur de s'épanouir dans l'acte d'écriture. Enfin, la dimension axiologique est teintée du milieu socioculturel du sujet (le milieu où il a grandi, les écoles et les enseignants qu'il a fréquentés), car ses représentations sociales se sont construites et développées sous l'influence de ce milieu.

La conceptualisation des dimensions de la compétence scripturale retenues a servi de cadre d'analyse pour cette étude. Les deux principaux concepts étant définis, nous présentons maintenant la méthodologie de la recherche. 


\section{Méthodologie}

Cette recherche qualitative descriptive visait à présenter un portrait des caractéristiques des difficultés reliées à la compétence scripturale d'enseignants débutants et à décrire les répercussions de ces difficultés sur leurs tâches et leur insertion professionnelles. Ainsi, nous souhaitions dresser un portrait, une représentation détaillée et fidèle des difficultés dans la compétence scripturale d'enseignants débutants du primaire et décrire les relations qui existent entre ces difficultés, les tâches professionnelles et l'insertion professionnelle de ces enseignants, ce qui, selon SavoieZajc (2011) et Angers (2009), renvoie à une recherche descriptive. Lors de la première partie de cette étude, nous avons obtenu des données quantitatives à propos des difficultés déclarées dans la compétence scripturale grâce à l'utilisation d'un questionnaire fermé. Puis, lors de la seconde partie de la collecte, nous avons réalisé des entrevues semidirigées afin de pouvoir décrire les répercussions des difficultés déclarées sur les tâches et l'insertion professionnelle des enseignants. Des méthodes mixtes ont donc été employées afin d'atteindre les objectifs de recherche.

\section{Échantillonnage}

Le questionnaire en ligne a été envoyé au printemps 2013 à tous les enseignants débutants du primaire des commissions scolaires de Laval, des Affluents et de la Seigneurie-des-Milles-Iles et a été dument rempli par 44 enseignantes. Nous avons ensuite réalisé des entrevues avec 11 d'entre elles qui témoignaient avoir des difficultés dans la compétence scripturale. Ces onze enseignantes étaient des femmes âgées de 20 à 30 ans qui avaient entre une et quatre années d'expérience. Huit d'entre elles avaient un contrat et elles enseignaient à différents cycles. Nous croyons que la taille de cet échantillon est suffisante pour faire ressortir les difficultés dans la compétence scripturale puisque, comme l'affirme Savoie-Zajc (2011), une quarantaine de participants peut permettre d'arriver à la saturation des données. Ces enseignants débutants devaient répondre au critère élaboré par Martineau et Ndoreraho (2006) qui est de se consacrer à temps plein à la carrière d'enseignant depuis moins de cinq ans. La participation était volontaire; un échantillonnage non probabiliste de convenance a donc été privilégié, puisqu'il « donne accès à une connaissance détaillée et circonstanciée de la vie sociale » (Poupart et al. 1997, p.97).

\section{Analyse des données}

Premièrement, les données recueillies par les questionnaires ont fait l'objet d'une codification selon le cadre théorique de la compétence scripturale et ont été transférées en format statistique à l'aide de l'outil intégré dans le logiciel Lime Survey. Elles ont été ensuite transférées dans le logiciel Excel afin d'être saisies dans un tableau à entrées multiples qui nous a fourni les proportions de réponses obtenues pour chaque question. Nous avons également dégagé des statistiques descriptives.

Deuxièmement, les données obtenues par les entrevues semi-dirigées ont été analysées selon la technique d'analyse de contenu (Van der Maren, 1996), puisque cette stratégie correspond bien au contenu symbolique d'une approche qualitative (L'Écuyer, 1990). Ce type d'analyse s'effectue selon cinq phases chronologiques : 1) déterminer les unités d'analyse; 2) élaborer un système de codes; 3) classer les différentes unités d'analyse sous les composantes du cadre théorique prédéfini; 4) synthétiser les données 
pour en faire une représentation graphique; 5) proposer une interprétation des données recueillies. Le logiciel $Q D A$ Miner a été utilisé pour analyser ces données qualitatives et nous avons principalement observé les fréquences de codage.

Quelques résultats. Nous présentons maintenant quelques résultats ressortis lors des analyses quantitatives et qualitatives.

\section{Difficultés dans la compétence scripturale}

Rappelons d'abord que notre premier objectif spécifique de recherche est de décrire les difficultés déclarées sur le plan de la maitrise de la compétence scripturale des participantes. Concernant les savoirs linguistiques de la compétence scripturale, les premiers résultats révèlent des aspects intéressants puisque $52 \%$ des participantes ont affirmé éprouver souvent ou très souvent des difficultés à suivre les règles orthographiques et grammaticales; $38 \%$ ont affirmé avoir souvent ou très souvent des difficultés avec la ponctuation; et $30 \%$ ont mentionné éprouver souvent ou très souvent des difficultés à trouver le ton qui convient selon le destinataire. Rappelons que les participantes au questionnaire n'ont pas été choisies parce qu'elles éprouvaient des difficultés dans la maitrise de la compétence scripturale.

En ce qui a trait aux réponses obtenues par le biais des entrevues semi-dirigées, réalisées uniquement auprès de répondantes ayant mentionné éprouver des difficultés dans la compétence scripturale, elles révèlent que toutes les participantes, lorsque nous leur avons demandé de préciser leurs difficultés, ont débuté en nommant des difficultés liées aux savoirs linguistiques. Les savoirs orthographiques, nommés en premier par les 11 participantes, représentent un défi pour ces enseignantes comme en témoigne E6 :

"J'ai de la misère avec l'orthographe: des mots comme salopette, je ne sais pas s'il faut que je mette un "l”, un " $p$ " ou 2 "l” ou 2 " $p$ ”. Si je dois écrire ce mot-là, il faut que je vérifie comment ça s'écrit. »

Le même constat peut être fait pour les règles grammaticales et la conjugaison qui ont été nommées par toutes les participantes. Quatre d'entre elles ont d'ailleurs mis en parallèle leurs difficultés grammaticales avec la préparation qu'elles ont effectuée pour réussir le test de certification en français écrit pour l'enseignement (TECFÉE), dont la réussite est obligatoire pour poursuivre des études dans tous les programmes de formation à l'enseignement des universités québécoises francophones. L'enseignante E5 résume bien les propos des enseignantes nouvellement qualifiées lorsqu'elle mentionne que :

«pour le TECFÉE, je me suis bourré le crâne de toutes les règles : bijou, caillou, les règles de couleurs, les exceptions de conjugaison... Le problème, c'est que je ne les ai pas appris [sic], je les ai momentanément mémorisées, je me rappelle donc de plein de bouts de règles, mais jamais des règles au complet. Je ne suis pas très avancée. »

Cet énoncé nous permet de comprendre l'étendue des difficultés que représentent les savoirs linguistiques et l'utilité perçue du TECFÉE cette nouvelle enseignante. De plus, deux participantes ont mentionné qu'elles auraient préféré pouvoir s'inscrire à une session de mise à niveau en grammaire avant de passer le test afin d'éviter les échecs et 
les effets néfastes sur leur estime de soi. Dans un autre ordre d'idées, une enseignante actuellement sous contrat en sixième année a abordé la grammaire de la phrase. Elle mentionne éprouver des difficultés à repérer les groupes de mots, les fonctions syntaxiques et à identifier les classes des mots. Nous pouvons donc penser que les difficultés dans les savoirs linguistiques influencent non seulement l'écriture des enseignants, mais aussi les notions grammaticales plus complexes qu'elles doivent de surcroit enseigner aux élèves, notamment quand elles sont au troisième cycle.

Sur le plan de la dimension affective, lorsque nous avons demandé aux répondantes dans le questionnaire « dans le cadre de votre travail, diriez-vous que vous aimez écrire? », $35 \%$ des participantes mentionnent qu'elles aiment un peu ou pas du tout écrire, alors que $54 \%$ des répondantes disent aimer assez écrire. Seulement $10 \%$ des participantes ont coché qu'elles aimaient beaucoup écrire. Nous devons rappeler que les répondantes au questionnaire n'éprouvaient pas nécessairement des difficultés dans la compétence scripturale.

Durant les entrevues, le code «affective » est ressorti 34 fois et jamais de façon positive. Ainsi, les 11 participantes aux entrevues, qui ont des difficultés dans la compétence scripturale, n'affectionnent pas l'écriture et témoignent toutes de leur sentiment négatif vis-à-vis de l'écriture. L'enseignante $\mathrm{E} 3$ résume bien la situation lorsqu'elle mentionne :

«Je n'aime pas écrire, mais j'aime enseigner. Le problème c'est que je n'avais pas pensé qu'en enseignement, il faut tellement écrire : écrire au tableau ce qui inclut le mot d'accueil le matin, pendant que j'enseigne, en faisant un remue-méninge, les mots pour les autres enseignantes, les messages aux parents, les réunions de profs... Ça, c'est les fois évidentes, il y a les sournoises aussi : la correction, écrire les commentaires dans les bulletins, je suis sûre que j'en oublie, mais on écrit tout le temps. Je pense que c'est quand on n'aime pas ça qu'on se rend compte à quel point il faut écrire. »

Finalement, les enseignantes E4 et E7 sont les seules à avoir traité du lien entre leurs difficultés dans la compétence scripturale et leur aversion pour l'écriture. L'enseignante E4 mentionne à cet effet :

"C'est rare qu'on aime vraiment quelque chose dans lequel on n'est pas bon. Si je suis vraiment poche en ski, ce ne sera surement pas mon sport préféré. Moi, je ne suis pas bonne en écriture et je n'aime pas écrire... C'est logique. "

Maintenant que les données à propos de la dimension affective ont été exposées, nous passons à la dernière dimension de la compétence scripturale, la dimension axiologique.

Pour la dimension axiologique, les répondantes au questionnaire devaient écrire, en quelques mots, ce que représentait l'écriture pour elles en termes de sentiment et de valeur. Concernant les sentiments, dix participantes ont utilisé exclusivement des termes positifs pour décrire l'écriture (valorisant, agréable, libérateur) tandis que quinze répondantes n'ont employé que des termes négatifs (contraignant, stressant, angoissant, 
souffrant). Il y a donc 11 répondantes sur 44 qui ont utilisé à la fois des termes positifs et négatifs pour décrire leur sentiment vis-à-vis de l'écriture. En ce qui a trait aux valeurs, elles mentionnent en grande majorité (90\%) que l'écriture est nécessaire, primordiale et très importante. On peut donc penser que les recrues connaissent l'importance de l'écriture dans leur profession, malgré les difficultés rencontrées.

\section{Répercussions sur les tâches professionnelles}

Les tâches professionnelles inhérentes à l'enseignement nécessitent souvent l'usage de l'écriture. Notre deuxième objectif spécifique de recherche, décrire les tâches professionnelles des enseignants débutants qui sont affectées par leurs difficultés déclarées dans la compétence scripturale, était intimement lié à ce constat. Les participantes aux entrevues semi-dirigées se préoccupent beaucoup de leurs difficultés dans la compétence scripturale, car ces dernières ont des répercussions sur leurs tâches professionnelles. Elles sont nombreuses à avoir mentionné des tâches telles que l'encadrement des élèves, la correction, la planification et l'enseignement magistral.

L'encadrement, c'est le suivi des élèves sur le plan du comportement. Pour les enseignantes interrogées, c'est surtout la communication aux parents qui s'avère laborieuse. En effet, toutes les nouvelles enseignantes ont relaté des répercussions de leurs difficultés sur la communication avec les parents et le code " encadrement » est revenu 100 fois sur 380 durant les entretiens. C'est le code qui est le plus présent dans les tâches éducatives.

La principale répercussion est le temps accordé à chaque message adressé aux parents. Les enseignantes débutantes affirment devoir passer beaucoup de temps à écrire ces messages. Même lorsqu'elles utilisent un support informatique, elles doivent se relire plusieurs fois et trois recrues mentionnent qu'elles ne sont jamais certaines qu'il ne reste plus d'erreurs lorsqu'elles envoient le message. Comme elles n'aiment pas écrire, neuf d'entre elles préfèrent appeler les parents le soir. Elles affirment toutefois se sentir débordées et avoir l'impression qu'elles ne décrochent jamais réellement. De plus, trois enseignantes qui préfèrent appeler les parents soutiennent que cette technique est également très longue, puisque les parents en profitent pour prendre des nouvelles à propos du développement de leur enfant, de ses relations sociales et de raconter des évènements qui se passent à la maison. L'enseignante E5 indique : «J'ai l'impression d'être dans un cul-de-sac : écrire les messages c'est vraiment long et passer mes soirées au téléphone, c'est mortel. » Malgré le fait que plusieurs préfèrent appeler les parents, certains parents préfèrent les messages dans l'agenda et les courriels, et certains enfants ont une feuille de route que l'enseignante se doit de remplir tous les jours.

Huit enseignantes signalent qu'elles ont peur que les parents trouvent des erreurs dans leurs messages. Elles estiment qu'une telle situation peut miner leur crédibilité auprès des parents, mais aussi auprès des élèves. Elles craignent également les représailles si un parent devait faire une plainte. À ce sujet, l'enseignante E9 a, à deux reprises, reçu son message de la veille signé par un parent qui avait encerclé une ou plusieurs erreurs orthographiques et grammaticales. Cette situation est terriblement stressante, voire embarrassante, pour elle qui souligne que :

"[...] les deux fois, j'ai tellement pleuré parce que je me sentais tellement mal et que j'avais peur que les parents fassent une plainte à la direction. 
J'ai été vraiment chanceuse, ils ne l'ont pas fait, mais c'est comme si je marchais sur des oufs avec eux. Je me dis que si je fais quelque chose qui ne leur plait pas, ils vont aller voir la direction pour parler de la situation et ils vont surement ramener sur le tapis que j'ai fait des fautes. C'est comme un stress qui est toujours là. Chaque fois que le directeur veut me voir, je me demande si c'est ça. »

Finalement, les messages aux parents sont une grande source de stress pour toutes les enseignantes interrogées lors des entrevues semi-dirigées. Lorsque nous leur avons demandé si les obstacles qu'elles vivent lors de la rédaction des messages aux parents étaient, en partie, dus à leurs difficultés dans la compétence scripturale, elles ont toutes répondu oui. Nous présentons maintenant quelques répercussions des difficultés sur l'insertion professionnelle.

\section{Répercussions sur l'insertion professionnelle}

Cette dernière section réfère au troisième objectif spécifique de cette étude, soit de décrire les répercussions des difficultés dans la compétence scripturale sur l'insertion professionnelle.

Sur le plan de la construction et de la consolidation des savoirs, les résultats obtenus montrent que les difficultés dans la compétence scripturale ne semblent pas accentuer les défis liés à la gestion de classe. En ce qui a trait aux savoirs liés à la pédagogie, une seule participante considère que ses difficultés affectent les techniques d'enseignement qu'elle utilise. À ce propos, l'enseignante E1 mentionne :

Au début, j'essayais de ne pas faire beaucoup d'enseignement magistral parce que je pensais que ce serait plus dur de ne pas faire de fautes, mais je me suis vite rendu compte que l'enseignement coopératif ou par projet est encore plus difficile à gérer. Tous les élèves écrivent des choses différentes et je dois corriger sur le vif, sans pouvoir utiliser mon iPhone ou Antidote. Ça modifie vraiment mes façons ou mes techniques d'enseignement.

Finalement, sept participantes émettent le constat que l'insertion est un moment très difficile et elles trouvent que leurs lacunes dans la compétence scripturale ajoutent des difficultés supplémentaires. Ainsi, l'enseignante E11 spécifie : «Je sais que l'insertion est dure pour pas mal tout le monde. Moi en plus, j'ai mes difficultés en français qui s'ajoutent. C'est la goutte qui fait déborder le vase. "

Pour ce qui est du rapport aux autres, six participantes ont mentionné que leurs difficultés nuisent aux rapports avec les élèves. Ces enseignantes ont peur que le fait de commettre des erreurs devant les élèves leur fasse perdre de la crédibilité. Trois de ces enseignantes débutantes font valoir que les relations entre enseignants et élèves sont souvent basées sur un sentiment de confiance mutuelle. Elles ont l'impression de briser ce lien de confiance et de décevoir les élèves lorsqu'elles commettent une erreur ou qu'elles doivent aller vérifier sur leur ordinateur portable une règle de grammaire ou l'orthographe d'un mot. L'enseignante E9 mentionne à cet égard que : "Les élèves s'en rendent compte, surtout les plus vieux, ils le voient bien que je cherche certains mots sur mon cellulaire. Ça mine ma crédibilité et j'ai l'impression qu'ils vont moins me faire 
confiance. " Sur le plan du rapport aux collègues et à la direction, toutes les enseignantes interviewées mentionnent qu'elles souhaitent cacher leurs difficultés à leurs collègues et à la direction de leur école. Elles vivent également avec la peur constante de se faire prendre par un collègue et elles doivent trouver des moyens pour pallier leurs difficultés afin de passer inaperçues. Cinq enseignantes mentionnent que, vu leur besoin de cacher leurs difficultés et la lourdeur des tâches professionnelles qui requièrent l'écriture, elles se sentent isolées et à l'écart des autres. L'enseignante E9 explique sa situation ainsi :

" J'aimerais ça me mêler plus à la vie de l'école, mais je travaille tout le temps. C'est comme si les autres enseignantes sont habituées que je ne sois jamais là. Elles ne me portent aucune attention. Je sais que c'est parce que je ne suis pas là, car il y a deux autres nouvelles à l'école et tout le monde leur parle beaucoup. Moi, je reste dans ma classe à corriger et à planifier et je ne me fais pas d'amies. »

Le sentiment d'isolement semble donc être amplifié par les difficultés dans la compétence scripturale et cela peut rendre le rapport aux autres plus laborieux. $\mathrm{Vu}$ le temps passé à corriger, à planifier et à élaborer les activités d'apprentissage, les enseignantes se disent très fatiguées, voire épuisées.

La dimension du développement de l'identité professionnelle est ressortie dans toutes les entrevues. À propos de la connaissance de soi en tant qu'enseignante, les participantes devaient s'évaluer à propos de leur compétence scripturale, en s'attribuant une note sur 10. Elles ont toutes mentionné leurs difficultés et en ont parlé en termes de limites, de défis, de problèmes, de lacunes, de gêne et d'embarras. Ainsi, l'enseignante E3 indique :

"Quand on enseigne, on écrit tout le temps, alors quand on a de la difficulté avec l'écriture et l'orthographe, on a un gros problème. Ça limite nos choix de techniques pédagogiques et ça nous met un stress terrible, non seulement pour ne pas se faire prendre, mais pour savoir quoi faire quand on se fait prendre. »

En outre, les onze participantes ont souligné que leurs difficultés dans la compétence scripturale avaient des répercussions sur leur estime de soi. L'enseignante E5 mentionne «à cause de mes difficultés en écriture, je me sens tellement poche, tellement pas à ma place. »

Comme nous l'avons exposé dans la section sur les tâches professionnelles, toutes les enseignantes ont souligné le stress qu'elles vivent quotidiennement afin de réaliser certaines tâches comme la planification et l'enseignement. À ce propos, le stress ressenti à l'égard de l'enseignement et le sentiment d'incompétence pédagogique sont tels que 10 enseignantes ne souhaitent enseigner qu'à des élèves du premier cycle. L'enseignante E5 explique l'enjeu de ce choix dans l'extrait suivant :

"Ce n'est pas un caprice, je ne peux pas enseigner aux élèves plus vieux. Si je me retrouve à avoir un contrat avec des cinquième ou sixième année, c'est 
sûr que je lâche. Je ne lâcherais pas juste le contrat, peut-être que je lâcherais tout court. »

Cette citation permet de constater que l'estime de soi négative pourrait aller jusqu'à affecter l'envie de persévérer dans la profession. Cette position a été adoptée par quatre enseignantes tandis que trois autres affirment rester en sachant qu'elles se sont peut-être trompées de carrière.

La dernière composante de l'insertion professionnelle, l'acquisition de la culture institutionnelle, englobe la connaissance de la clientèle, les normes et les règles du milieu, les habitudes et les pratiques pédagogiques des collègues, la philosophie et les valeurs du milieu. Les données analysées montrent que les difficultés dans la compétence scripturale ne semblent pas amplifier les défis que vivent les enseignantes dans l'acquisition de la culture institutionnelle en contexte d'insertion professionnelle.

\section{Discussion}

Nous proposons de clore cet article en effectuant une courte discussion des résultats présentés et en envisageant quelques perspectives pour des recherches futures.

En ce qui a trait aux difficultés dans la compétence scripturale, Lefrançois et al. (2005) mentionnent que certains étudiants en enseignement ont des difficultés marquées en écriture, particulièrement sur les plans de l'orthographe lexicale et grammaticale. Nos données corroborent ce constat, car $52 \%$ des répondantes au questionnaire rapportent éprouver des difficultés considérables en orthographe lexicale et grammaticale. En fait, lorsque nous examinons les données recueillies avec le questionnaire, il est possible d'affirmer que plus de $40 \%$ des répondantes témoignent avoir de grandes ou de très grandes difficultés à maitriser les savoirs linguistiques (orthographe, grammaire, ponctuation, syntaxe). Ce constat a également été effectué lors des Etats généraux sur la situation et l'avenir de la langue française au Québec alors que nous y apprenions que la moitié des futurs enseignants québécois ne possédaient pas, à cette époque, une maitrise de la langue française suffisante pour exercer leur fonction (Larose, 2001). Les résultats de notre étude abondent dans le même sens puisque, tel que présenté dans les résultats, les difficultés dans la compétence scripturale rendent difficile la réalisation de certaines tâches professionnelles.

D'ailleurs, en ce qui concerne les répercussions sur les tâches professionnelles, il semble que les difficultés dans la compétence scripturale amplifient leur lourdeur. Mukamurera et al. (2008) affirment que la tâche lourde et difficile est le principal motif d'abandon de la profession ou de remise en question chez les enseignants débutants en général. Nos résultats correspondent à ce constat et toutes les participantes ont parlé de la lourdeur de la tâche et du sentiment d'être débordées, voire envahies par toutes ces tâches. Dans la même recherche, $40 \%$ des participants affirmaient se sentir souvent dépassés par la charge de travail. Quant à nos résultats de recherche, ce sont toutes les enseignantes qui ont effectué ce constat. Nous avons pu constater que le fait d'avoir des difficultés dans la compétence scripturale contribue à rendre les tâches professionnelles des enseignantes débutantes encore plus difficiles à réaliser et accentue leur sentiment d'être dépassées et débordées. Dans le même ordre d'idées, la charge de travail élevée et le manque de temps peuvent être des sources de stress, d'insomnie et de dépression chez les nouveaux enseignants (Martineau \& Vallerand, 2005). Les participantes ont parlé 
d'épisodes de tristesse et de colère à l'égard de leurs difficultés et des tâches qu'elles doivent accomplir. Plus particulièrement, le fait de prendre beaucoup de temps pour corriger et planifier semble très stressant pour elles.

Finalement, en ce qui concerne les retombées des difficultés sur l'insertion professionnelle, Martineau (2006) soutient que les enseignants débutants associent souvent leurs difficultés à une formation initiale de piètre qualité. Encore une fois, les données recueillies dans le cadre de notre recherche corroborent l'idée que les enseignantes débutantes jugent que la formation initiale est inadéquate ou insuffisante. Ajoutons que certaines enseignantes ont déploré ne pas avoir reçu plus d'aide pour leurs difficultés en français lors de leur parcours universitaire et auraient aimé avoir un cours de grammaire. Pourtant, plusieurs universités offrent des services d'aide en français personnalisés et gratuits, ainsi que des formations gratuites pour la préparation au TECFÉE. Il y a également des cours de didactique de la grammaire et de didactique de l'écriture dans tous les BEPEP. Nous considérons donc important de nous questionner sur ce que les participantes ont entrepris, par elles-mêmes, afin d'aller chercher l'aide nécessaire pour s'améliorer durant leur parcours universitaire et durant leur insertion professionnelle. Les stratégies les plus employées pour surmonter les difficultés sont l'utilisation de correcteurs informatiques et l'évitement. Nous constatons que les participantes jugent sévèrement la formation initiale et souhaitent obtenir un cours de trois crédits sur la grammaire, mais elles ne semblent pas être enclines à participer aux formations gratuites demandant un engagement supplémentaire de leur part.

De plus, nous savons que les difficultés vécues par les enseignants débutants lors de leur insertion professionnelle peuvent avoir des effets négatifs sur leur estime de soi (Presseau \& Martineau, 2007). Les enseignantes participantes ont toutes soulevé ce point et jugent que leurs difficultés dans la compétence scripturale nuisent à leur insertion professionnelle et à leur estime personnelle. Elles se disent stressées par leurs difficultés, car ces dernières amplifient le stress et la charge de travail qui sont déjà des facteurs de risque importants pour les enseignants en général (Leroux, 2010).

\section{Conclusion}

Notre recherche s'inscrit dans une optique de prévention, en ce sens qu'il est important de prendre conscience que les difficultés dans la compétence scripturale peuvent nuire à la réalisation des tâches professionnelles et à l'insertion professionnelle. Les résultats générés par notre recherche pourront avoir des retombées sur la formation initiale et continue, sur les programmes d'insertion professionnelle et sur les programmes universitaires, en suscitant notamment une réflexion sur le TECFÉE, sur sa pertinence. En effet, une réflexion s'impose à savoir si la passation du TECFÉE garantit que les futurs enseignants possèdent les compétences nécessaires dans la compétence scripturale et s'il serait nécessaire d'ajouter d'autres mesures. De plus, nous devons réfléchir aux moyens d'amener les étudiants à se responsabiliser lorsqu'ils éprouvent des difficultés dans la compétence scripturale, en les sensibilisant aux répercussions possibles et aux multiples tâches professionnelles qui nécessitent l'écriture. Finalement, les résultats de notre recherche pourraient influencer le soutien accordé par les directions d'école aux enseignants nouvellement qualifiés en les conscientisant sur les difficultés dans la compétence scripturale et sur l'attention à porter à ces difficultés lors de leurs évaluations. 


\section{Références}

Barré-De Miniac, C. (2000). Le rapport à l'écriture: aspects théoriques et didactiques. Lille, France : Presses universitaires du Septentrion.

Beckers, J. (2007). Compétences et identités professionnelles : l'enseignement et autres métiers de l'interaction humaine. Bruxelles, Belgique : De Boeck.

Bédard, Y. (2000). L'insertion professionnelle du jeune enseignant par la méthode de "mentoring» (Mémoire de maitrise inédit). Université du Québec en AbitibiTémiscamingue.

Boucher, M. (2001). Aux âmes bien nées... : portrait de trois jeunes enseignantes. Vie pédagogique, 118, 9-10.

Centrale des syndicats du Québec (2005). Les différentes voies d'accès menant à la profession enseignante. Québec, Québec : CSQ.

Chartrand, S.G. (2000). Le programme de français de 1995. Un outil pour développer la compétence d'écriture au secondaire. Québec français ( $n^{\circ}$ hors série) : La grammaire au cour du texte, 24-27.

Chartrand, S.G., \& Blaser, C. (2008). Du rapport à l'écriture au concept didactique de capacités langagières à écrire et à lire : apports et limites de la notion de rapport à l'écriture. Dans S.-G. Chartrand \& C. Blaser (dir.), Le rapport à l'écrit(ure), un outil pour penser les situations didactiques. Namur, Belgique : Dyptique.

Curtis, K. C. (2005). An Analysis of the Construct of Efficacy on New Teacher Retention (Thèse de doctorat inédite). George Mason University.

Dabène, M. (1987). L'adulte et l'écriture : contribution à une didactique de l'écrit en langue maternelle. Bruxelles, Belgique : De Boeck Université.

Fédération des syndicats de l'enseignement du Québec. (2011). Les droits, pouvoirs et responsabilités des enseignantes et des enseignants.

Hayes, J. R. \& Flower, L. S. (1980). Identifying the organization of writing processes. Dans L.-W. Gregg \& E.-R. Steinberg (dir.), Cognitive processes in writing. Hillsdale, $\mathrm{NJ}$ : Lawrence Erlbaum Associates.

Larose, G. (2001). Le français, une langue pour tout le monde. Une nouvelle approche stratégique et citoyenne. Québec, Québec : Gouvernement du Québec.

Lefrançois, P., Laurier, M.D., Lazure, R., \& Claing, R. (2005). Évaluation de l'efficacité des mesures visant l'amélioration du français écrit du primaire à l'université. Rapport de recherche. Montréal, Québec : Université de Montréal.

Leroux, M. (2010). Étude des relations entre la résilience d'enseignantes et d'enseignants du primaire cuvrant en milieux défavorisés et la réflexion sur la pratique (Thèse de doctorat inédite). Université de Montréal.

Martineau, S. (2006). À propos de l'insertion professionnelle en enseignement. Formation et Profession, 12(2), 48-54.

Martineau, S., \& Bergevin, G. (2007). Le mentorat. Trois-Rivières, Québec : Université de Québec à Trois-Rivières.

Martineau, S., \& Mukamurera, J. (2012). Tour d'horizon des principaux programmes et dispositifs de soutien à l'insertion professionnelle en enseignement. Revue Phronesis, l(2), 45-62.

Martineau, S., \& Ndoreraho, M.A. (2006). Une problématique des débuts de carrière en enseignement. Université de Québec à Trois-Rivières. Repéré à http://www.insertion.qc.ca/cnipe_2/IMG/pdf/JPN_ET_SM-2.pdf. 
Martineau, S., \& Presseau, A. (2003). Le sentiment d'incompétence pédagogique des enseignants en début de carrière et le soutien à l'insertion professionnelle. Brock Education, 12(2), 54-67.

Martineau, S., \& Vallerand, A.-C. (2005, décembre). L'insertion professionnelle des enseignants : un enjeu pour le milieu scolaire, un défi pour le monde de la recherche. Conférence présentée au séminaire du CRIFPE, Jouvence, Québec.

Martineau, S., \& Vallerand, A.-C. (2006). Que peuvent faire les directions d'école pour favoriser l'insertion professionnelle des nouveaux enseignants? Formation et profession, 13(1), 43-48.

Maurais, J. (1999). La qualité de la langue: un projet de société. Québec, Québec: Conseil de la langue française.

Ministère de l'Éducation du Québec (2001). La formation à l'enseignement - les orientations et les compétences professionnelles. Québec, Québec : Gouvernement du Québec.

Mukamurera, J., Bourque, J. \& Gingras, C. (2008). Portraits et défis de l'insertion en enseignement au Québec pour les nouvelles générations d'enseignants. Dans L. Portelance, J. Mukamurera, C. Gervais \& S. Martineau (dir.), L'insertion dans le milieu scolaire: une phase cruciale du développement professionnel de l'enseignant (49-72). Québec, Québec : Les Presses de 1’Université Laval.

Mukamurera, J., Martineau, S., Bouthiette, M., \& Ndoreraho, J.P. (2013). Les programmes d'insertion professionnelle des enseignants dans les commissions scolaires du Québec : portrait et appréciation des acteurs. Education \& Formation, 299, 1-23.

Norman, J. P., \& Feiman-Nemser, S. (2005). Mind activity in teaching and mentoring. Teaching and Teacher Education, 21, 679-697.

Presseau, A., \& Martineau, S. (2007). L'évaluation des apprentissages dans un environnement scolaire marqué par la différenciation pédagogique et l'enseignement en faveur du transfert: quelques résultats d'une recherche-action menée avec des enseignants du primaire. Actes du $19^{e}$ colloque ADMÉE-Europe, 1(1), 1-3.

Schneuwly, B. (1995). Apprendre à écrire. Une approche sociohistorique. Dans J.-Y. Boyer, J.-P. Dionne \& P. Raymond (dir.), La production de textes. Vers un modèle d'enseignement de l'écriture (p. 73-100). Montréal, Québec : Les Éditions Logiques.

Simard, A. (2005). L'insertion professionnelle au secondaire : Un défi à relever. ÉCHO du R.É.S.É.A.U. Laval, Bulletin des écoles associées à l'Université Laval, 5(1), 19-21.

Vallerand, A.-C. (2008). Mobilisation de stratégies chez les enseignants en insertion professionnelle (Mémoire de maitrise inédit). Université du Québec à Trois-Rivières.

Van der Maren, J.M. (1996). Méthodes de recherche pour l'éducation. Montréal, Québec : Les Presses de l'Université de Montréal.

\section{Biographie de L'auteur}

Geneviève Carpentier est détentrice d'une maitrise en éducation de l'Université du Québec en Outaouais (UQO). Ses intérêts de recherche concernent principalement l'insertion professionnelle des enseignants et la didactique du français. Elle est chargée de cours à l'UQO où elle offre notamment des cours reliés à la didactique du français. 